\title{
Special Section Guest Editorial: Recent Advances in Lens Design and Optical Engineering
}

\author{
Simon Thibault, ${ }^{\text {a }}$ R. Barry Johnson, ${ }^{\mathrm{b}}$ Joseph Mulley, ${ }^{\mathrm{c}}$ and \\ Cornelius Hahlwegd \\ ${ }^{a}$ University Laval, Québec, Canada \\ ${ }^{b}$ Alabama A\&M University, Normal, Alabama, United States \\ 'IDEX Health \& Science, LLC, Rochester, New York, United States \\ dbbw Hochschule-University of Applied Sciences, Berlin, Germany
}

Over nearly four decades, we have seen an increase in the impact of optical technologies in our daily lives. This remarkable progress is due to major development in optical science and engineering. Optical engineering is a fascinating activity, ranging from operational instruments to lens design and modeling with the help of immensely powerful design software. Beyond optical design, the optical engineer is concerned with the fabrication of components, assembly and alignment techniques, metrology and calibration, as well as the interaction with other engineering disciplines such as mechanical, thermal, electronic, and software. This special section offers the most recent results of the multi-faceted discipline that is lens design and optical engineering, and the multi-talented individuals that dedicate themselves to this field. The lens designer and the optical engineer, often the same person, will find this special section a place to stay abreast of the frontiers of this constantly evolving field. Overall, the special section consists of a dozen papers that cover various aspects of optical engineering, from zoom lenses to optomechanics.

Two papers deal with the constantly evolving field of zoom lenses. They show how optical modeling, fabrication, and mechanical design have, in their own specific ways, furthered the methods of designing these systems as well as continued to expand their performance capabilities and therefore applications. Another paper highlights some advances made in passively aligning lenses. These new methods can open up new design-space possibilities and tradeoffs for the optical designer to take advantage of.

Three papers contribute to the field of Fourier optics and optical systems theory in a broader sense. One contribution is dedicated to extended depth-of-field imaging using diffractive optical elements with special consideration to achromatic behavior. Another one in the field of computational imaging solves the problem of overcoming limited sampling rates by application of a workpiece rotating technique for the in situ measurement of micro-structured surfaces, leading to extraction of accurate depth information for such structures. A third paper explores the field of digital holographic microscopy, introducing an improved adaptive spatial filter method for extraction and interpretation of parts of the hologram spectrum.

In the paper titled "Analytic equations to design optical systems with three stigmatic pairs in the meridional plane," authors Rafael Guillermo González-Acuña, Héctor A. Chaparro-Romo, and Julio C. Gutiérrez-Vega present closed-form analytical expressions to design lens systems comprising an arbitrary number of refractive lenses that are sigmatic axially and for two symmetric off-axis points. Actually, since this is a rotationally symmetric optical system, it arguably should be thought of as axially sigmatic and a stigmatic ring of object points having a constant radius. The authors provide an illustrative example of their method. The elegance of their method is that it is based fundamentally on Snell's law and Fermat's principle, which exclude any optimization or numerical approximation. Now that free-form surfaces are practical to fabricate, it will be interesting to see how expansively the authors can further develop closed-form analytical expressions to design more complex lens systems.

An interesting discussion of the linear astigmatism aberration is presented by José M. Sasián and Weichuan Gao in their paper titled "Characteristics and control of linear astigmatism aberration in a nonaxially symmetric optical system," which offers a perceptive approach to

(C) 2021 Society of Photo-Optical Instrumentation Engineers (SPIE) 
appreciate how linear astigmatism originates based on stop shifting in the presence of uniform coma aberration. The paper provides a comprehensive study of linear astigmatism in a clear and orderly manner as well as an in-depth discussion of formulas to quantify and correct linear astigmatism. Various examples of conic-mirror type optical systems corrected for linear astigmatism are discussed.

Literally of billions of miniature lenses have been designed and built for various electronic devices, such as smartphones and tablets. Rather common specifications for these lenses are $\mathrm{F} / 2.8$, total track length of $4 \mathrm{~mm}$ to $6 \mathrm{~mm}$, and FOV ranging from about $20^{\circ}$ to $80^{\circ}$. These lenses are almost always made of plastic today and the number of elements range typically from four to six. Due to the use of very high-order aspheric coefficients, the surface shape of some lenses has one or more inflection points. In the paper "Improving as-built miniature lenses that use many aspheric surface coefficients with two desensitizing techniques," authors Leticia Carrión-Higueras, Arnau Calatayud, and José M. Sasian discuss two approaches to desensitize this type of lens to errors in tilt and decentration. The first approach is to gradually reduce the number of high-order aspheric coefficients while achieving the performance goals. The second approach optimizes the unperturbed configuration and several perturbed configurations at the same time. Both techniques were found to work for improving as-built lenses. Examples are discussed in detail for a four-element lens system and a six-element lens system. For those interested in the design of lens systems containing surfaces with high-order aspheric coefficients, this paper should be added to your reading list.

The paper from Haiyin Sun, who has 30 years of experience in optics and lasers, focuses on the high power density stripe of diode laser. "On the focusing of wide stripe laser diode astigmatic beams for highest power density" discusses how to focus these wide stripes of diode astigmatic beam as small as possible. The discussion includes alignment and how it must be properly controlled to reduce the astigmatism of the focused beam.

Finally, freeform compact design is still a hot topic these days. A team from the National Institute of Astrophysics, Optics, and Electronics (INAOE), Mexico, presents a new design of an off-axis three mirror system that is corrected for spherical, coma, astigmatism, and field curvature. The author provides a step-by-step process starting from paraxial theory to obtain a compact, lightweight, easy-to-align optical system. Remaining aberration is then compensated by freeform, and a diffraction limited system is obtained. 